\title{
Nosocomial spread of COVID-19: lessons learned from an audit on a stroke/neurology ward in a UK district general hospital
}

\author{
Authors: Soraya V Jewkes, ${ }^{A}$ Yimeng Zhang, ${ }^{\mathrm{B}}$ and David J Nicholl'
}

We describe the details of a COVID-19 outbreak in a 25-bedded Birmingham neurology/stroke ward in the early phase of the pandemic (March to May 2020). Twenty-one of 133 admissions (16\%) tested positive for COVID-19 and of those, 8 ( $6 \%$ of all admissions to the ward) were determined to be nosocomial. Thus 38\% (8/21) of COVID-19 infections were hospital-acquired. Ten of the patients that contracted COVID-19 died; of these three were hospital-acquired cases. Five of the 21 patients had negative swabs prior to receiving a positive test result. This study highlights the importance of appropriate use of personal protective equipment (PPE) with high-risk patients (including those with stroke and complex brain injury with tracheostomies) and the difficulties of COVID-19 management in a high-risk patient population.

KEYWORDS: COVID-19, nosocomial infection, stroke, neurology, infection control

DOI: $10.7861 /$ clinmed.2020-0422

\section{Introduction}

Coronavirus disease 19 (COVID-19) has presented new challenges globally. The West Midlands has been one of the UK areas worst hit by the serious acute respiratory syndrome coronavirus 19 (SARS-CoV-2) pandemic.

Although many steps are taken to protect inpatients from COVID-19, difficulties can arise, such as the availability of siderooms and the logistics of inpatient social distancing, which has been shown to minimise spread.?

This audit was conducted after noticing a trend of COVID-19 diagnoses in patients who could not have contracted SARS-CoV-2 from outside the hospital or from visitors. This was during the initial phase of the pandemic when $19.2 \%$ of those being tested in the UK were positive for COVID-19. ${ }^{2}$

Authors: ${ }^{A}$ core medical trainee, Sandwell and West Birmingham NHS Trust, Birmingham, UK; Binternal medicine trainee, Sandwell and West Birmingham NHS Trust, Birmingham, UK; 'clinical lead for neurology, Sandwell and West Birmingham NHS Trust, Birmingham, UK
We identified both spatial and temporal links between positive cases, with not only patient-patient but also patient-staff transmission. This highlights the importance of good hygiene and appropriate use of personal protective equipment (PPE). The intention is not to find fault, but to learn. Such lessons can then be applied during any future outbreak, enabling a faster change in daily practice for the benefit of both staff safety and patient care.

\section{Methodology}

Data were collected retrospectively for all patients admitted onto a 25-bed acute stroke ward over a 54-day period during the initial phase of the pandemic (12 March 2020 to 5 May 2020). This was deemed to be a 'cold' ward, ie not specifically designated for suspected COVID-19 cases. Data were collected on patient demographics and risk factors for contracting SARS-CoV-2 from the trust's electronic patient record system. The bed movements of all patients subsequently testing positive for COVID-19 (based on their viral reverse-transcription polymerase chain reaction [RT-PCR] swab) were tracked along with date of test and result.

We also collected data from consenting ward staff with regular patient contact regarding their need for isolation and PCR results. Staff members such as porters, imaging technicians and phlebotomists were not tracked due to the difficulty in identifying individuals and their minimal periods of direct contact. Hospitalacquired COVID-19 infection is defined as a positive PCR result seen in a patient who had no clinical suspicion of disease on admission and who developed symptoms more than 14 days after admission to hospital.

This audit was registered and approved within the Trust (ref. SC1193).

\section{Results}

A total of 133 patients were admitted to the ward during the study period. Of these, 21 (15.8\%) went on to test positive for COVID-19; this was not significantly different to the UK data for this period $(19.2 \%$; $p>0.05) .{ }^{2}$ Of those, eight $(6 \%)$ were determined to be nosocomial. Consequently 38\% (8/21) of COVID-19 infections were hospital-acquired in this ward, a significantly greater percentage than was seen in the Trust overall (5.2\% [52/999]; $\mathrm{p}<0.00001)$. Patient demographics are summarised in Table 1. Ten of the patients that contracted COVID-19 died, of which three 
Table 1. Patient demographics

$\begin{array}{lll}\text { Total COVID-19 positive, } \mathrm{n} & & 21 \\ \text { Sex, } \mathrm{n}(\%) & \text { Male } & 12(57) \\ & \text { Female } & 9(43) \\ \text { Age, years } & \text { Mean } & 75 \\ & \text { Range } & 45-96 \\ & \text { Median } & 73 \\ \text { Ethnicity, } \mathrm{n}(\%) & \text { Not stated } & 2(10) \\ & \text { Asian } & 3(14) \\ & \text { Black } & 2(10) \\ & \text { Chinese } & 1(5) \\ & \text { White } & 12(57) \\ & \text { Other } & 1(5)\end{array}$

were hospital-acquired cases. Five of the 21 patients had negative swabs prior to receiving a positive test result. In addition, two patients were identified as having had two tests done on the same day and received both a positive and a negative result. Our COVID19-positive patients had on average been moved to four (range 1-8) inpatient bed spaces during the study period, not including the emergency department (ED) and acute medical unit (AMU).

\section{Discussion}

\section{Hospital-acquired COVID-19}

Public Health England (PHE) has estimated the rate of nosocomial infections to be $10-22 \%,{ }^{3}$ whereas a meta-analysis of Chinese data estimated numbers $44 \%{ }^{4}$ Our rate of nosocomial infection exceeds PHE's estimation and is also significantly higher than Trust-wide rates within our own Trust (5.2\%). Reasons for this are likely multifactorial, with many identified aspects specific to our department and patient population. We have discussed some of the significant elements below.

\section{Staffing/ward layout}

Like many hospitals, the majority of beds are arranged in bays with a small number of side-rooms, as outlined in Fig 1. Each bay accommodates five or six patients, with hatched areas indicating a nurses' station. There is one door to enter/exit the ward, making the creation of a one-way flow system impossible. The work areas for staff are small with no specific donning and doffing areas.

Side rooms shared nursing staff with bays. Due to the specialist input needed, patients were by preference isolated in side-rooms rather than being moved to another ward. As a result, patients with confirmed or suspected COVID-19 in side-rooms were cared for by the same staff looking after patients with low or no suspicion in the bays. The use of dedicated staffing for 'hot' and 'cold' areas may not be sustainable with limited staffing, and there may be challenges with the creation of separate work and rest areas.

The first COVID-19 case on the ward was a stroke patient who became symptomatic within 4 days of admission during the early phase of the pandemic in the UK (Patient A, Fig 1a). They deteriorated suddenly and the emergency response team was called to the bedside twice in the 24 hours before transfer to the intensive treatment unit (ITU) for intubation. Both times the attending staff were not wearing any PPE as per local and national guidelines at the time. Nineteen nursing staff were symptomatic following contact with this patient, of which 15 subsequently swabbed positive. However, given the high community prevalence of COVID-19 during this period, not all of these staff will have had nosocomial infections. ${ }^{2,5}$

There was a high rate of ward staff sickness and confirmed COVID-19 infections (Table 2). Not all symptomatic staff were swabbed or self-isolated. Self-isolation protocol at the time did not classify anosmia or mild symptoms in the criteria. One nurse had 'indeterminate' symptoms and two COVID-19 swabs were lost. None of the speech and language team had to self-isolate or required swabbing. This was attributed to early adoption of PPE including visors and disposable respirators due to their high risk of direct exposure.

Box 1 outlines the much more rigorous infection control measures, including quarantine for 14 days, that have been used successfully in other countries such as in South Korea, and which should be considered essential. ${ }^{6}$

\section{Bed movements}

During this period, all admitted patients were allocated to either a 'hot' possible COVID-19, or 'cold' COVID-19-free pathway, and then assigned to respective wards. The acute stroke ward remained a 'cold' ward throughout the audit period, with side rooms used to isolate suspected or known COVID-19-positive patients.

A patient suspected of having an acute stoke would generally be seen in the ED. From there, if they were not thought to have COVID-19, they were moved to the acute stroke unit. Many patients were then transferred from here to the stroke-rehabilitation ward. Neurology patients were admitted via a similar route, or admitted to hospital straight from home or repatriated from another trust for on-going neurorehabilitation. Medical outliers were also admitted to the stroke ward when available, through the ED and AMU. These pathways result in most patients being transferred to at least two or three wards. However, following through the movements of the COVID-19 positive patients, it is clear that patients have a high movement within wards as well as between wards (Fig 1a,b). This is secondary to a combination of clinical need and bed management coordination; however, this inevitably increased the risk of transmission of COVID-19 to and from other patients and staff. This is demonstrated in Fig 1c, which follows the movement of patients over one day. Patient A, highlighted in red, moved bed space three times in a 24 -hour period. He received a positive COVID-19 test the subsequent day. In addition to this, the patient had moved between the rehabilitation ward to the acute ward and back again in the previous 48 hours, totalling six bed spaces shared in a 5 -day period. The movement between wards was due to patient A becoming acutely unwell; however, this, and also the frequent movement between bays, meant potential exposure of neighbouring patients and multiple staff members to COVID-19. In addition, patient B, whose COVID-19 test returned positive the next day, was transferred to an external bed. Patient $C$ was moved into the bed space occupied by Patient A and then into patient B's bed space the following day. It should be noted that the picture only illustrates the movement of patients that become COVID-19 positive; not seen are the multiple bed moves that occur in the patients that never test positive or become symptomatic. 
a) 16 March 2020
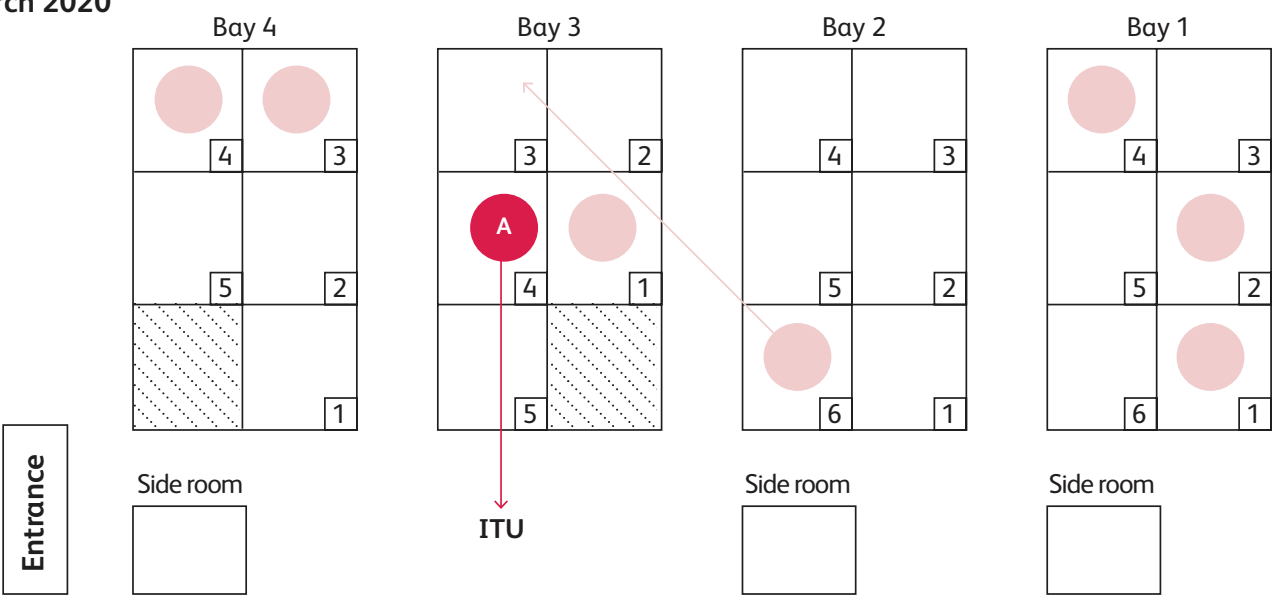

b) 23 March 2020
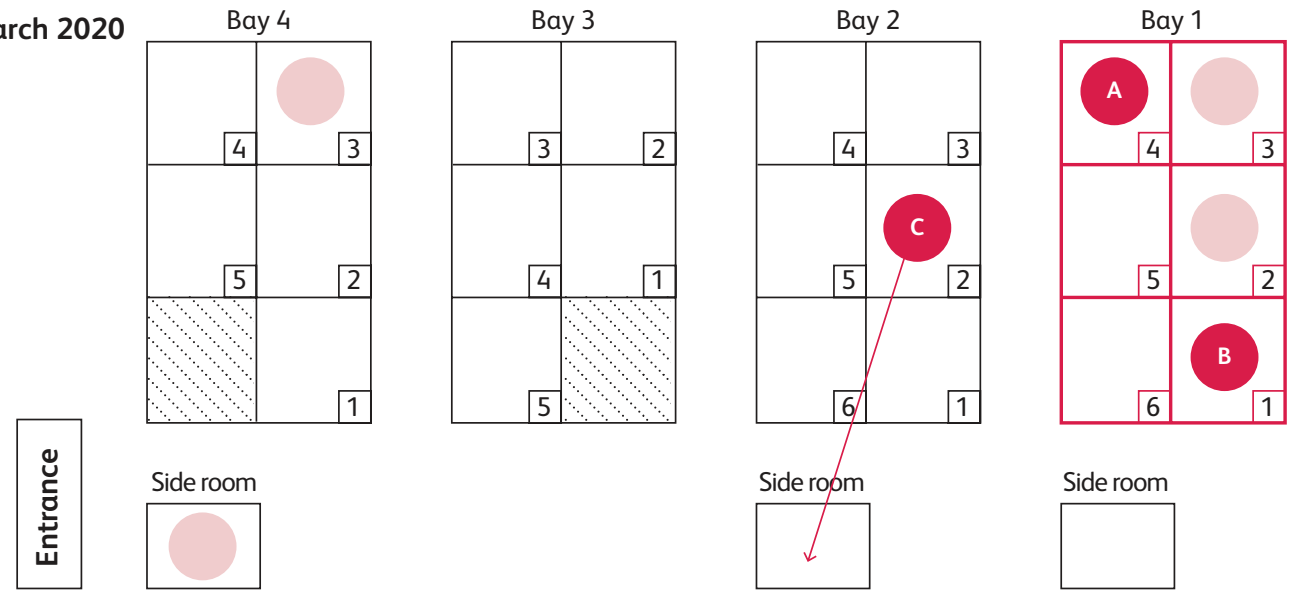

c) 31 March 2020
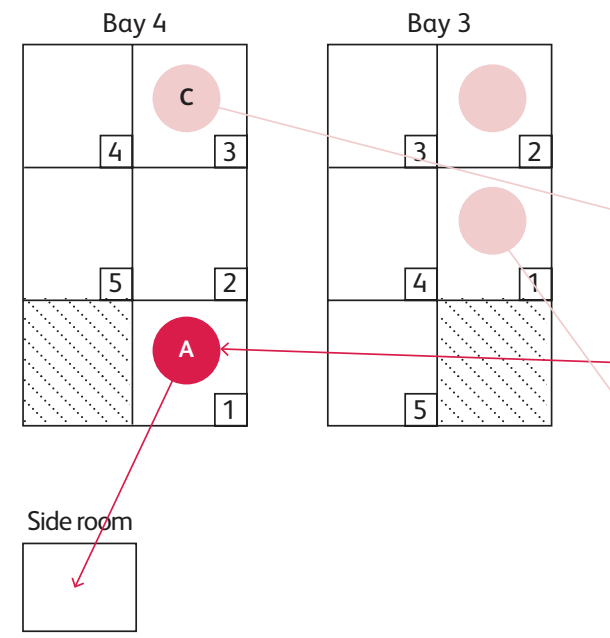

Bay 2

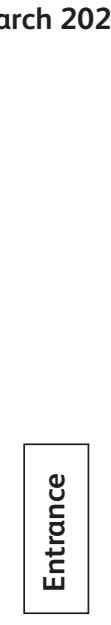

Key:

Patient not currently symptomatic

Patient movement

Symptomatic patient

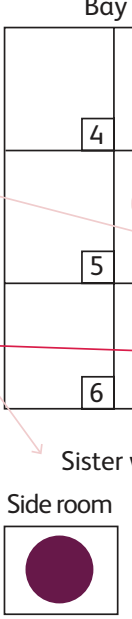

Bay 1

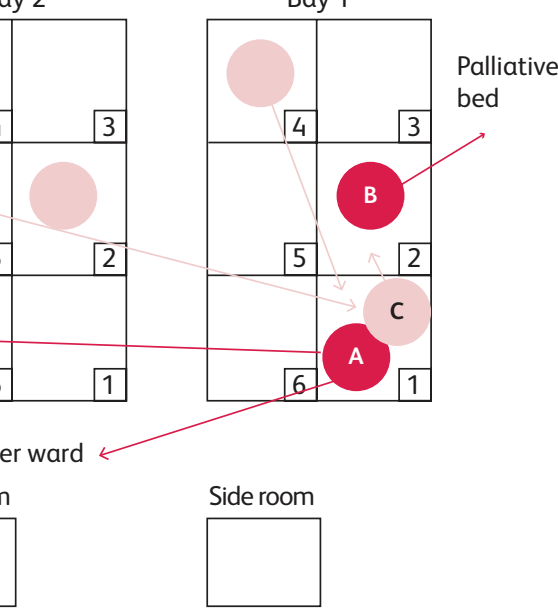

Fig 1. Ward layout and the movements of inpatients on three selected days in March 2020. All patients shown are either COVID-19-positive or will test positive later in the admission. a) 16 March. Patient A was the first patient on the ward to later test positive for COVID-19. The patient moved into the bay indicated and the patient opposite both later went on to test positive. b) 23 March. The UK entered full lockdown. Two patients became symptomatic on this day. As a result, the entire bay was isolated and all patients swabbed until the status of all patients were known. Patient B tested positive, while the other three tested negative. They would all go on to test positive later in their admissions. Patient $C$ also became symptomatic and was isolated in a side room. c) 31 March. Multiple patient moves occurring on one day. Notably, patient A moved bed space three times in a 24-hour period, only to receive a positive COVID-19 test the subsequent day. 
Table 2. Staff illness

$\begin{array}{lllllll} & \text { Ward administrator, } & \text { Therapy team, } & \text { Healthcare assistant, } \\ \mathbf{n = 4} & \mathbf{n = 1 7} & \mathbf{n = 1 4} & \begin{array}{l}\text { Nurse, } \\ \mathbf{n}=\mathbf{3 0}\end{array} & \begin{array}{l}\text { Junior doctor, } \\ \mathbf{n}=\mathbf{1 5}\end{array} & \begin{array}{l}\text { Consultant, } \\ \mathbf{n}=\mathbf{7}\end{array} \\ \text { Isolation due to symptoms, } \mathrm{n}(\%) & 2(50) & 9(52) & 6(43) & 19(63) & 9(60) & 3(43) \\ \text { Positive swab PCR, } \mathrm{n}(\%) & 1(25) & 6(35) & 5(36) & 13(43) & 3(20) & 2(29) \\ \text { No isolation, } \mathrm{n}(\%) & 2(50) & 8(48) & 8(57) & 11(36) & 6(40) & 4(57)\end{array}$

\section{PCR swabs}

In the early phase of the outbreak, testing was difficult for a number of reasons. The first tests available were not only scarce, but had a high false negative rate, and to an extent testing also carried with it a stigma. In the very early phase of the pandemic there was a reluctance to test suspected cases due to the fear this would create among both staff and patients. This in turn contributed to a delay in diagnosis and appropriate isolation.

The policy for testing for RT-PCR during the audited period involved nasal and pharyngeal swabs only from patients with a clinical syndrome suggestive of infection. At the beginning of the pandemic, staff PCR testing was not available. Hospital staff exhibiting symptoms of fever or persistent cough were offered a nasopharyngeal swab from 18 March depending on availability and advised on isolation accordingly.

Our suspected false negative rate of $33 \%$ was higher than those identified in other studies; however, variations are known to occur due to suboptimal sampling techniques, the primer used for the sample and the timing of the sample in relation to patient disease

Box 1. Lessons learned from Sandwell COVID-19 outbreak: future measures to help us 'respond like South Korea' ${ }^{\prime}$

> Universal testing and intensive screening and management of high-risk facilities in vulnerable groups (eg nursing home residents, patients with complex neurology)

> Isolation of any suspected COVID-19 case, until two negative samples (preferably deep respiratory samples) with complete resolution of symptoms

$>$ Cohorting of exposed contacts and monitoring for symptoms for 14 days

> Social distancing (2-metre rule in non-clinical areas) during handover meetings and in staff areas

> Self-quarantine of all staff with close contact with a COVID-19 patient with any breach of PPE or with positive staff member in non-clinical area where social distancing not adhered to

> Prioritise infection control over capacity issues

> Fast-turnaround, high sensitivity COVID-19 testing available $24 / 7$

$>$ Awareness of false negative rates

$>$ Universal mask coverage

$>$ Meticulous tracheostomy care (whatever COVID-19 status) in a separate area

> Full PPE for any AGP (including if a patient is on humidified oxygen)

$>$ Visors for nasogastric tube insertion

$>$ Enhanced cleaning on a COVID ward with frequent cleaning of touch points (every 2 hours) process. A significant difference in the sensitivity of the PCR result has been noted between nasopharyngeal and oropharyngeal samples. ${ }^{7-9}$ This also contributed to uncertainty regarding the validity of negative results at a ward level.

The COVID-19 PCR swab results took $1-5$ days to return. There were several instances identified where suspected patients were swabbed and remained in a mixed bay, potentially exposing other patients and staff while awaiting results. For example, in one 4-day period, an entire bay was isolated after two patients became symptomatic and were swabbed; only one test came back positive initially, but the three other patients subsequently went on to test positive (Fig 1b). While this is out of the control of the ward team, changes could be made to the way patients are cared for while their results are pending. This should involve isolation in a side room or separate area.

Establishing precise modes of transmission would require a phylogenetic analysis of COVID-19 swabs, which would be hard to do given the numbers of cases in the UK, compared with smaller outbreaks in countries which have managed community transmission more successfully, such as in South Africa $^{10}$ or Hong Kong. ${ }^{11}$

\section{Vulnerable groups}

As this was a stroke/neurology ward, the majority of patients fell into a high or moderate-risk category due to their age, comorbidities or immunosuppressant use. Most patients had acute stroke and many were highly dependent. A significant proportion of the neurology patients were also highly dependent and clinically complex with a tracheostomy. In addition to this, aerosolgenerating procedures as part of tracheostomy care are regularly carried out in shared patient areas. Prior to COVID-19 they were carried out by staff without PPE and in the early stages using only fluid-resistant surgical masks.

Although the numbers are small, we suspect tracheostomy patients are an especially high-risk group, both from their propensity to catch COVID-19 and their risk of spreading it. As part of their routine care they undergo airway suctioning multiple times a day and frequent inner tube changes, both of which are now listed as aerosol-generating procedures and therefore present a high transmission risk. The tracheostomy patients in this cohort all had significant neurological injury and as a result were bedbound and unable to communicate. Signs of systemic infection such as raised temperature, tachycardia and tachypnoea are difficult to differentiate from the normal fluctuations resulting from their brain injury.

Overall, as others have noted, patients requiring neurorehabilitation may be an especially vulnerable group. We suggest that there needs to be regular Trust- and ward-level data available on COVID-19 prevalence to assist in targeting measures locally to combat the disease, ${ }^{12-14}$ as well as screening of healthcare staff as others have suggested. ${ }^{5}$ 


\section{Conclusion}

This is a study at the early stage of the UK COVID-19 pandemic in a ward of especially vulnerable patients, which is why we suspect the proportions for nosocomial infections is higher than those reported nationally. There were specific risk factors, including close nursing, therapy contact, tracheostomy and immunocompromised patients in a region with high disease prevalence in the community. Nosocomial infection cannot easily be prevented; however, rigorous infection control, handwashing and the use of appropriate PPE can reduce transmission. ${ }^{15}$

Several areas have been highlighted where significant improvements can realistically be made to reduce the rate of nosocomial transmission not only of COVID-19, but of any infection spread by respiratory droplets (Box 1). This is of particular significance as we do not know how long the pandemic will continue, with the possibility that COVID-19 diagnosis will become part of the new normal. Early adoption of adequate PPE is vital to protecting both patients and staff from nosocomial transmission. Other simple measures include keeping patient movement to a minimum. The high risk of contraction and asymptomatic transmission of COVID-19 among tracheostomy patients supports their routine isolation, not only to protect themselves but also the surrounding patients. This includes the continued use of full PPE by nursing staff when carrying out tracheostomy care. Given the wide variations in nosocomial COVID-19 rates in different environments, we would urge that all healthcare providers publish their COVID-19 rates, as they are likely to vary significantly depending on the patient population and care environment. Such transparency will make it much easier to develop more targeted approaches to deal with the pandemic. We would suggest that PHE looks urgently at our suggested measures, adapted from those used in South Korea, including 14-day quarantine.

\section{Acknowledgements}

We are grateful to all the members of staff who assisted in this audit. Special thanks to Salman Sadiq and Michael Brennan, and especially to our deceased patients' families.

\section{References}

1 Wee LE, Conceicao EP, Sim XY] et al. Minimising intra-hospital transmission of COVID-19: the role of social distancing. J Hosp Infect 2020;105:113-5.

2 Coronavirus (COVID-19) daily statistics (UK). Data from 12 March to 5 May 2020. https://covid19-uk.co.uk [Accessed 16 June 2020].
3 Harding L, Campbell D. Up to $20 \%$ of hospital patients with COVID-19 caught it at hospital. The Guardian, 17 May 2020. www.theguardian.com/world/2020/may/17/hospital-patientsengland-coronavirus-covid-19 [Accessed 4 ]une 2020].

4 Zhou Q, Gao Y, Wang X et al. Nosocomial infections among patients with COVID-19, SARS and MERS: a rapid review and meta-analysis. MedRxiv 2020, 2020.04.14.20065730.

5 Treibel TA, Manisty C, Burton M et al. COVID-19: PCR screening of asymptomatic health-care workers at London hospital. Lancet 2020;395:1608-10.

$6 \mathrm{Kim} \mathrm{JH}, \mathrm{An}$ JAR, Min P et al. How South Korea responded to the COVID19 outbreak in Daegu. NEJM Catalyst 2020, in press (doi: 10.1056/CAT.20.0159).

7 Carver C, Jones N. Comparative accuracy of oropharyngeal and nasopharyngeal swabs for diagnosis of COVID-19. Centre for Evidence-Based Medicine, 2020. www.cebm.net/covid-19/ comparative-accuracy-of-oropharyngeal-and-nasopharyngealswabs-for-diagnosis-of-covid-19/.

8 Fang $\mathrm{Y}$, Zhang $\mathrm{H}$, Xie ] et al. Sensitivity of chest CT for COVID-19: comparison to RT-PCR. Radiology 2020, in press (doi: 10.1148/ radiol.2020200432).

9 Yang Y, Yang M, Shen C et al. Laboratory diagnosis and monitoring the viral shedding of 2019-nCoV infections. MedRxiv 2020, 2020.02.11.20021493.

10 Lessells R, Moosa Y, De Oliveira T. Report into a nosocomial outbreak of coronavirus disease 2019 (COVID-19) at Netcare St. Augustine's Hospital. KRISP, 2020. www.krisp.org.za/ manuscripts/StAugustinesHospitalOutbreakInvestigation FinalReport_15may2020.pdf [Accessed 4 June 2020].

11 Wong SY, Kwong RS, Wu TC et al. Risk of nosocomial transmission of coronavirus disease 2019: an experience in a general ward setting in Hong Kong. J Hosp Infect 2020;105:119-27.

12 Mamo J, Feroz B, Mahmood S. Covid-19: protecting patients in hospital for neurorehabilitation and their therapists. $B M J$ 2020;369:m1630.

13 McNeary L, Maltser S, Verduzco-Gutierrez M. 2020. Navigating coronavirus disease 2019 (Covid-19) in physiatry: A CAN report for inpatient rehabilitation facilities. PM R 2020;12:512-15.

$14 \mathrm{Li} \mathrm{N}$, Liu T, Chen $\mathrm{H}$ et al. Management strategies for the burn ward during COVID-19 pandemic. Burns 2020;46:756-61.

15 Thomas JP, Srinivasan A, Wickramarachchi CS et al. Evaluating the national PPE guidance for NHS healthcare workers during the COVID-19 pandemic. Clin Med 2020;20:242-7.

Address for correspondence: Dr David Nicholl, Department of Neurology, Birmingham City Hospital, Sandwell and West Birmingham NHS Trust, Dudley Road, Birmingham B18 7QH, UK. Email: david.nicholl@nhs.net 